\title{
Formación inicial de un profesorado inclusivo: avances y perspectivas en la carrera de pedagogía en educación física, deporte y recreación de la Universidad Austral de Chile
}

\author{
Initial training of an inclusive teaching staff: Progress and \\ perspectives in the physical education teaching program from \\ Austral University of Chile \\ Formação inicial de um corpo docente inclusivo: avanços e \\ perspectivas na carreira pedagógica em educação física, \\ esportes e recreação da Universidad Austral de Chile
}

Mauricio Alejandro Miranda Nahuelquin

Licenciado en educación Universidad Austral de Chile mauricioalejandromira@gmail.com

Pierre Richard Médor Magíster en educación mención política y gestión educativas

Universidad Austral de Chile pmedor1981@gmail.com

\section{Resumen}

En la presente investigación se pretende analizar las competencias docentes en estudiantes de la carrera de Pedagogía de Educación Física, Deporte y Recreación de la Universidad Austral de Chile para el manejo de personas con necesidades educativas especiales en contextos educativos formales. Esta inquietud nace debido a otra investigación que revela una escasa preparación en la formación de los estudiantes de Pedagogía en Educación Física de la Universidad Austral de Chile, quienes expresan un desconocimiento de los términos y de cómo tratar con personas en situación de discapacidad. El enfoque de la investigación es el interpretativo, cimentado en la metodología de investigación cualitativa, y el diseño es de carácter Fenomenológico. El instrumento empleado en el estudio es la entrevista semiestructurada, validada por un experto, y que fue grabada para 
un mejor entendimiento y comprensión de cada uno de los sujetos. Los resultados nos indican que se puede evidenciar un avance en la carrera de Pedagogia en Educación Física, Deporte y Recreación de la Universidad Austral de Chile, debido al nuevo optativo impartido en la carrera.

Palabras claves: Formación Inicial, Inclusión, Pedagogía en Educación Física, Integración, Necesidades Educativas Especiales.

\begin{abstract}
The present research intends to analyze the teaching competences in students of the Pedagogy of Physical Education, Sports and Recreation course of the Austral University of Chile for the management of people with special educational needs in formal educational contexts. This concern was born due to another investigation that reveals a scarce preparation in the formation of the students of Pedagogy in Physical Education of the Austral University of Chile, who express a lack of knowledge of the terms and how to deal with people in a situation of disability. The focus of the research is the interpretative one, based on the methodology of qualitative research, and the design is of Phenomenological character. The instrument used in the study is the semi-structured interview, validated by an expert, and recorded for a better understanding and understanding of each of the subjects. The results indicate that an advance in the career of Pedagogy in Physical Education, Sport and Recreation of the Austral University of Chile can be evidenced, due to the new elective taught in the race.
\end{abstract}

Keywords: Initial Training, Inclusion, Pedagogy in Physical Education, Integration, Special Educational Needs.

\title{
Resumo
}

Nesta pesquisa é analisar as competências pedagógicas nos alunos da Pedagogia de Educação Física, Esporte e Lazer Universidade Austral do Chile para lidar com pessoas com necessidades educativas especiais em contextos educativos formais. Esta preocupação surge porque a outra pesquisa que revela uma falta de preparação na formação de estudantes de Pedagogia em Educação Física da Universidad Austral de Chile, que expressam ignorância dos termos e como lidar com as pessoas com deficiência. $\mathrm{O}$ foco da pesquisa é o interpretativo, baseado na metodologia da pesquisa qualitativa, e o design é de caráter fenomenológico. O instrumento utilizado no estudo é a entrevista semiestruturada, validada por um especialista, e registrada para melhor compreensão e compreensão de cada um dos sujeitos. Os resultados indicam que um avanço na carreira de Pedagogia em Educação Física, Esporte e Recreação da Universidade Austral do Chile pode ser evidenciado, devido à nova eletiva ensinada na prova. 
Palavras-chave: Formação Inicial, Inclusão, Pedagogia em Educação Física, Integração, Necessidades Educativas Especiais.

\section{Introducción}

La investigación planteada pretende indagar sobre la formación del profesorado de Educación Física y Salud, y el estado de su preparación en función de los desafíos que pueden surgir en el manejo de las necesidades educativas especiales en contextos escolares. El grupo por investigar es la generación 2014 de la carrera Pedagogía en Educación Física, Deporte y Recreación de la Universidad Austral de Chile.

La posibilidad de acceder a la educación ha sufrido algunos cambios en Latinoamérica gracias a diversas entidades como la UNESCO o la Organización de las Naciones Unidas, además de las declaraciones que dan apoyo a una educación más inclusiva como lo son la Declaración de Salamanca y la de Jomtien (1990). La inclusión se ha enmarcado como el medio más eficaz para combatir las actitudes discriminatorias, contribuir a la construcción de una sociedad y lograr la educación para todos (UNESCO, 1990). Además, existen diversos investigadores que se refieren a una educación de calidad y para todos como eje central de la inclusión, entre ellos Blanco (2006), Ainscow y Echeita (2011) y Ainscow (2015), quienes presentan unas ideologías similares con la educación para todos que consiga formar una sociedad más justa e igualitaria.

En lo que respecta a la carrera de Pedagogía en Educación Física, Deporte y Recreación, diversos estudiantes han tratado de investigar cómo se relaciona la inclusión con la preparación del profesorado y las políticas públicas, lo que ha dejado al descubierto que, aun cuando se han creado nuevas políticas o proyectos educacionales, estos ayudan a que los estudiantes lleguen al aprendizaje esperado dejando de lado la atención a la diversidad, donde es el estudiante quien se acomoda a la educación y no la educación a las necesidades de los estudiantes (Gallardo y Ríos, 2013). Por otro lado, en la formación de los profesores de Educación Física y Salud en la Universidad Austral de Chile existe un déficit en lo que respecta al conocimiento de la inclusión; así fue manifestado por los profesores en formación quienes participaron en la elaboración de una tesis:

Manifiestan desconocimiento de conceptos y terminologías sobre educación inclusiva, para personas con estado de discapacidad (...) se suma la insatisfacción e inseguridad en su formación inicial docente, lo que conlleva a un desempeño incierto (...) Esto se le atribuye al hecho de que la carrera no posee asignaturas específicas relacionadas con la atención a la 
diversidad, atención a las necesidades educativas especiales, educación física adaptada, educación física para personas con discapacidad. Siendo un tema importante en el contexto escolar y en el desarrollo como docente integral (Vásquez, 2013, p.70).

En consideración a lo anterior, surge la necesidad de revisar el estado actual de la preparación docente en estudiantes de la generación 2014 de Pedagogía en Educación Física, Deporte y Recreación frente a temas de inclusión educativa.

En la población meta se consideró a algunos de los estudiantes que cursaron por un nuevo optativo implantado en la malla de la carrera: "Motricidad Adaptada y Deportes Adaptados", en donde se entregan metodologías para poder trabajar con personas con necesidades educativas especiales, además del conocimiento de conceptos como inclusión, integración, exclusión y segregación. Además, aquellos que cursaron el ramo de "Practica de Integración. Ámbito Salud y/o Social" en donde los designan en diferentes centros integrales. Después de haber pasado por estos módulos tendrán una interpretación más clara con respecto a la malla de la carrera: si existe alguna falencia o vacío que deba mejorar para poder lograr una educación de calidad.

\section{Antecedentes}

En la indagación de investigaciones relacionadas con la formación del profesorado, este expresa una inconformidad en la formación de docentes para responder a la heterogeneidad de estudiantes que hoy en día es común evidenciar en las aulas de clases de los colegios. Lo que da cuenta a reforzar o incorporar en las mallas curriculares de las Universidades que imparten pedagogía, sus procesos formativos en atención a la diversidad (Ortiz, 2007; Tenorio, 2011).

Otras de las barreras existentes que expresan las investigaciones son la realización de la clase, donde no se cuenta con los materiales necesarios para poder trabajar con todo el grupo; la infraestructura no es la adecuada, ya que algunos colegios no cuentan con gimnasio -las opciones son pasillos más amplios o patios, en los que en días de lluvia suelen aparecer charcos de agua o piedrecillas. De este modo se dificulta poder responder a estudiantes con necesidades educativas especiales y, en general, la inclusión educacional (Mendoza, 2008; Infante, 2010).

La investigación llevada a cabo, busca averiguar cómo podría ser la preparación de un profesorado inclusivo, teniendo en cuenta los avances y las perspectivas de la carrera de Pedagogía en Educación Física, Deporte y Recreación de la Universidad Austral de Chile. Por 
lo tanto, se hizo primordial realizar una búsqueda de las investigaciones de generaciones anteriores, para dar a conocer si ya existía algún trabajo similar o si tal vez hubiese algún vacío con respecto al tema a investigar.

Una de las investigaciones realizadas en la carrera busca cuestionar las políticas públicas, tomando en consideración el Proyecto educativo institucional de ciertos colegios y los Programas de Integración Educacional, para revelar si en realidad estos conceptos ayudan a la inclusión y a responder a las necesidades de los y las estudiantes sin hacer discriminación alguna. Lo que reveló fue que a pesar de que ciertamente hay una ayuda en la integración, aún tiene un déficit en la inclusión (Gallardo y Ríos, 2013) debido a que lo que se plantea es una ayuda para que los educandos puedan cumplir con los planes y programas, haciendo que ellos se adecuen a los objetivos. Lo anterior evidencia un déficit de la respuesta a la diversidad de estudiantes que puedan existir en los colegios, mientras que lo que debería de suceder es que los programas se adecuen a la diversidad $y$ necesidades de los y las alumnas.

Otra investigación, que aborda desde la experiencia de la formación inicial docente y su interacción con personas en situación de discapacidad en los distintos espacios educativos, considerando la formación del Profesorado de Educación Física y Salud en la Universidad Austral de
Chile, dejó claro que la malla curricular de dicha universidad en aquel entonces tenía un déficit en la preparación de los futuros docentes para responder a las necesidades de todos sus estudiantes, haciendo énfasis en que no cumplen con las competencias para poder trabajar con personas en situación de discapacidad. Además, se manifestó un desconocimiento de los conceptos y terminologías sobre inclusión (Vásquez, 2013).

Las propuestas curriculares deben construirse sobre la base del desarrollo de competencias profesionales, expresadas en actitudes, conocimientos, metodologías y estrategias que permitan al profesorado dar atención de calidad a la diversidad educativa en la educación regular, considerando la diversidad como

una característica de la conducta y condición humana que se manifiesta en el comportamiento y modo de vida de los individuos, así como en sus modos de pensar y que se hace presente en todos los niveles evolutivos de la vida y en todas las situaciones. (Gutiérrez, 2004 en Ortiz, 2007, p. 47)

La educación como derecho está constantemente en boca de quienes trabajan la inclusión, pero con una intencionalidad de que esta educación sea equitativa y de calidad sin discriminar las capacidades de los educandos, aunque existen los desafíos académicos donde el currículo es muy estricto para el docente o no 
posee las herramientas necesarias para llevar a cabo una clase para todos sus estudiantes (Blanco, 2006).

Se ha visto en Chile que en las salas de clases regulares participan estudiantes en situación de discapacidad y estudiantes de otras nacionalidades que se han criado en este país, evidenciando el concepto de educación para todos sin causar una segregación. También el movimiento de la inclusión ha motivado la construcción de políticas de educación especial, el desarrollo de un programa intercultural y la construcción de asistencias técnicas para ayudar en la formación de los educandos (Infante, 2010). La intención enfatizada es que todos los estudiantes puedan aprender juntos en sistemas educativos abiertos que garanticen el desarrollo de las capacidades esenciales para la plena participación de la vida social y pública (Ortíz, 2007; Mendoza, 2008; Tenorio, 2011).

Para poder lograr una educación de calidad e inclusiva, se han manifestado diversas conferencias, decretos, con un largo historial en que se pueda llevar a cabo esta visión.

Tenemos igualmente la meta que queremos alcanzar, como quedó de manifiesto en la Conferencia Mundial de Salamanca (UNESCO, 1994). Además, la comunidad internacional ha otorgado el carácter de derecho a esta aspiración de una educación más inclusiva, como lo acaba de refrendar Naciones Unidas (2006): "para el caso de alumnado con discapacidad, (...) hay voluntad política refrendada en múltiples reuniones, conferencias y congresos, al más alto nivel, para avanzar en esa dirección, la inclusión" (UNESCO, 1994 en Echeita y Ainscow, 2011, p. 31).

Debido a la falta de preparación de las escuelas comunes, los padres y apoderados consideran como mejor opción llevar a sus hijos con necesidades educativas especiales a centros integrales en donde están seguros de que habrá profesionales capacitados para responder a las necesidades de sus hijos e hijas, además de las herramientas necesarias y la infraestructura. Aunque los deseos de los padres sean lo mejor para sus hijos, al llevarlos a un centro distinto al tradicional causan de forma inmediata una discriminación hacia ellos al separarlos de los demás estudiantes (Blanco, 2006). Lo otro que suele suceder es que, aquellos estudiantes que han ingresado al sistema regular desde instituciones especiales suelen mantenerse relativamente aislados del resto de sus compañeros. Incluso se denominan alumnos de "integración" o "inclusión" en lugar de ser tratados como alumnos "comunes" o "nuestros alumnos" (Ainscow, 2015). 


\section{Metodología}

El enfoque usado en la siguiente investigación es el interpretativo cimentado en la metodología de investigación cualitativa, debido a que en este tipo de investigación se "intenta interpretar y comprender la conducta humana desde los significados e intenciones de los sujetos que intervienen en la escena educativa" (Schuster, Puente, Andrada, y Maiza, 2013, p. 121).

El enfoque cualitativo tiene diversos tipos de estudios; en otras palabras podríamos decir que esta metodología "no constituye, pues, un enfoque monolítico sino un espléndido y variado mosaico de perspectivas de investigación" (Patton, 2002, p. 272 en Vasilachis, 2013, p. 2). Su desarrollo prosigue en diferentes áreas, cada una de las cuales está caracterizada por su propia orientación metodológica y por sus específicos propuestos teóricos y conceptuales acerca de la realidad, entre las más destacadas según Flick (2002) están:

1) La teoría fundamentada. 2) La etnometodología y el análisis de la conversación, del discurso y del género. 3) El análisis narrativo. 4) La hermenéutica objetiva y la sociología del conocimiento hermenéutica. 5) La fenomenología y el análisis de pequeños mundos de la vida. 6) La etnografía. 7) Los estudios culturales. 8) Los estudios de género (Flick citado en Vasilachis, 2013, p. 2).
De este modo se podrá explorar en la carrera de Pedagogía en Educación Física, Deporte y Recreación de la Universidad Austral de Chile la formación de los futuros docentes con respecto a la inclusión educacional.

El diseño es de carácter Fenomenológico debido a que "en el estudio, se pretende describir y entender los fenómenos desde el punto de vista de cada participante y desde la perspectiva construida colectivamente" (Hernández, Fernández y Baptista, 2006). En este caso se pretende comprender la formación del profesorado de Educación Física y Salud analizando y comprendiendo a los futuros docentes desde una mirada colectiva.

Para la muestra "nos conciernen casos (participantes, personas, organizaciones, eventos, animales, hechos, etc.) que nos ayuden a entender el fenómeno de estudio y a responder a las preguntas de investigación.

(Hernández, Fernández, y Baptista, 2006, p. 562). Debido a lo anterior se utiliza una "muestra teórica o conceptual" en donde "se eligen a las unidades porque poseen uno $o$ varios atributos que contribuyen a desarrollar la teoría" (Hernández, Fernández, y Baptista, 2006, pag. 569). Las personas elegidas para poder participar en esta investigación serán los/as estudiantes de cuarto año de la carrera de Pedagogía en Educación Física, Deporte y Recreación de la Universidad Austral de Chile, específicamente aquellos/as que 
aprobaron la asignatura de Motricidad Adaptada y Deporte Adaptado; debido al reducido número que aprobó el optativo (10) se pretende trabajar con todos/as, para lograr una mejor comprensión de la formación del profesorado de Educación Física y Salud.

La técnica empleada para recolectar los datos es una entrevista semiestructurada, ya que "se basan en una guía de asuntos $o$ preguntas $y$ el entrevistador tiene la libertad de introducir preguntas adicionales para precisar conceptos u obtener más información sobre los temas deseados (es decir, no todas las preguntas están predeterminadas)" (Hernández, Fernández, y Baptista, 2006, p. 597). El instrumento comprende nueve preguntas relacionadas con los objetivos específicos: tres preguntas para cada uno. Para la forma de aplicación se coordinó con cada participante y la entrevista fue grabada para una mejor comprensión de las respuestas. Esta entrevista fue validada por un experto.

Para que una investigación sea ética debe tener valor, lo que representa un juicio sobre su importancia social, científica o clínica; debe plantear una intervención que conduzca a mejoras en las condiciones de vida o en el bienestar de la población, o que produzca conocimiento que pueda abrir oportunidades de superación o de solución a problemas, aunque no sea en forma inmediata (González, 2002, p.98).

Como ya se ha expresado en los capítulos anteriores la justificación del presente informe es de carácter educativa con respecto a la formación del profesorado inclusivo: observar los avances y las perspectivas de la carrera Pedagogía en Educación Física, Deporte y Recreación. Los resultados ayudarán a revisar meticulosamente la malla curricular para una formación docente de calidad; no serán de inmediatos, pero con el tiempo se lograrán las mejoras.

A los/as sujetos que participaron en este proyecto de tesis se entregó un consentimiento informado que expresa el objetivo del trabajo, la garantía del anonimato y la confidencialidad, que la participación de cada uno/a es voluntario/a, no tendrá ningún aporte monetario hacia su persona, ni que corren algún tipo de riesgo. Lo anterior queda registrado con la firma de los informantes.

Se empleó la teoría fundamentada: el análisis de una palabra, frase u oración, debido a que esta técnica es especialmente valiosa, puesto que permite al analista plantear preguntas sobre posibles significados, supuestos o buscados. También puede despertar en el analista suposiciones acerca de lo que se dice o se observa, mientras se demuestra a sí mismo que existen otros posibles significados o 
interpretaciones (Strauss y Corbin, 2002, p. 116).

De esta manera con ayuda del software -el cual permite marcar las frases o palabras que están relacionadas con el estudio y otorgarle un nodo- podemos crear mapas conceptuales más fáciles, rápidos y bien relacionados. Es aquí donde estarán las categorías preestablecidas y aparecerán las categorías emergentes, aquellas de las que no esperábamos adquirir saberes, pero que pueden enriquecer el informe.

\section{Resultados}

Los resultados son expuestos según los objetivos específicos mencionados en la investigación para su mejor entendimiento: caracterizar la percepción de estudiantes en torno a las estrategias de formación en educación inclusiva a lo largo de su formación universitaria.

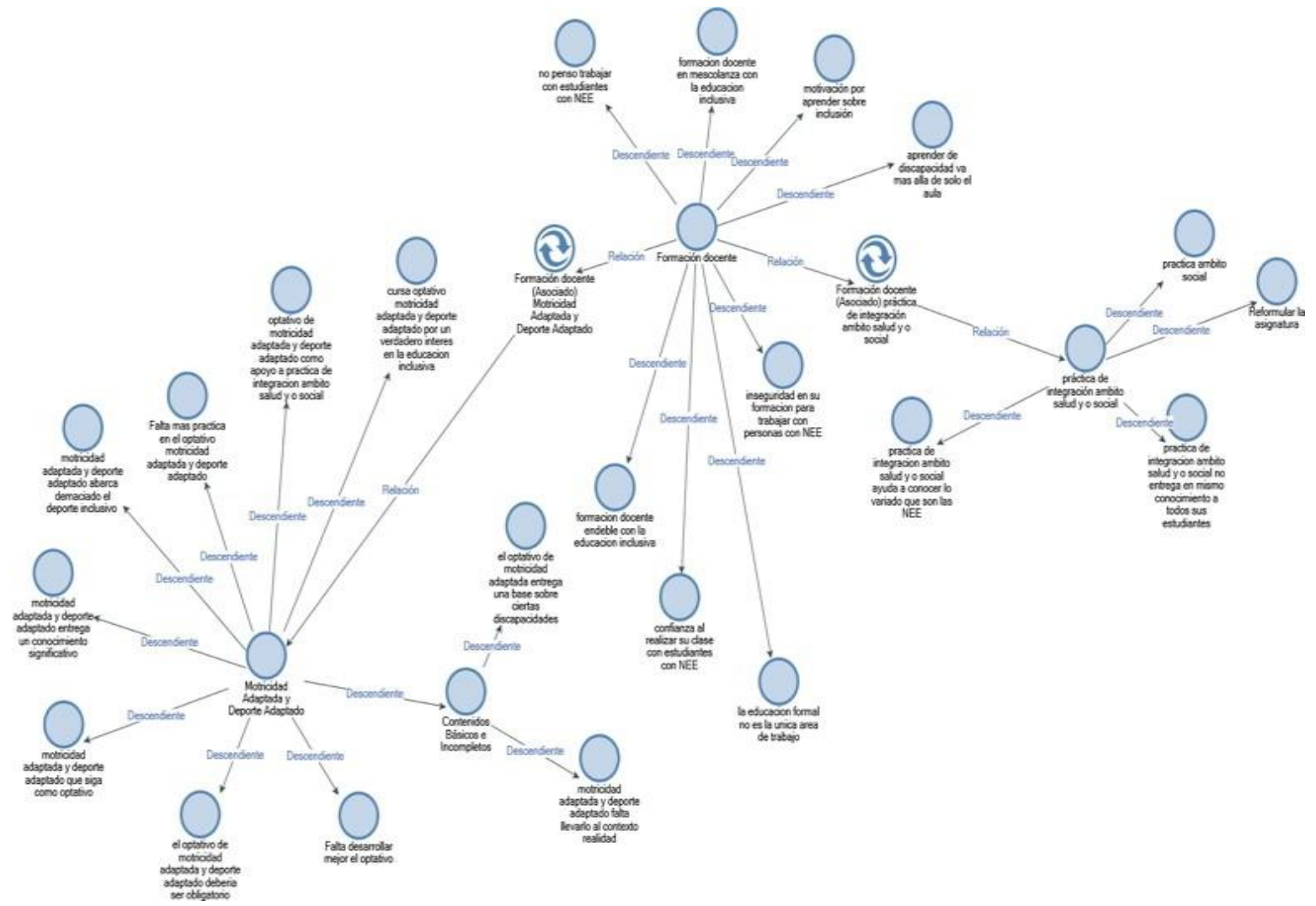

Figura 1 Pie: Intereses en la formación docente - Fuente: elaboración propia

Lo que se considera en la Figura 1 es la apreciación que tienen los estudiantes de la generación 2014 de Pedagogía en Educación Física, Deporte y Recreación de la Universidad Austral de Chile. Destaca que algunos estudiantes creen que la formación del profesorado debería ser en conjunto con la educación inclusiva y no tan solo que enseñen sobre este tema al final de la carrera -"yo creo que esto debería estar desde el día uno que uno 
ingresa a la Universidad" (sujeto 3, 2017, p. 5)- sino en todo el transcurso de esta. También "cuando a ti se te forma respecto a la educación inclusiva tú tienes más gamas de posibilidades de llegar a todo tipo de estudiantes" (sujeto 9, 2017, p. 6). Además destacan que el aprender sobre discapacidad va más allá de solo el sistema educativo y que debería ser un tema entendido por todos y todas: un trabajo que deben tener los padres y madres, los y las docentes, amigos/as, parientes y todas aquellas personas con las que conviven las personas en situación de discapacidad.

$\begin{array}{ccr}\text { Las } & \text { apreciaciones } & \text { que } \\ \text { entregaron } & \text { los } & \text { sujetos }\end{array}$ entrevistados también señalan que a pesar de tener asignaturas en las que les enseñan a cómo tratar con personas con algún tipo de discapacidad, destacan que aún les falta reformularlas de mejor forma y que a pesar de entregarles algunas herramientas y estrategias, los contenidos aún son muy básicos e incompletos en algunos casos. Es aquí donde las opiniones fueron divididas, donde algunos sujetos expresan que se sienten con seguridad en realizar una clase donde estén inmersos estudiantes con NEE y, por otro lado, algunos mencionan que sienten temor, nerviosismo e inseguridad al realizar una clase donde haya estudiantes con algún tipo de discapacidad.

A continuación, algunos de los resultados obtenidos con respecto a los efectos en la formación alcanzados en las modificaciones curriculares sobre participación, en las asignaturas de Motricidad Adaptada y Deportes Adaptados y la Práctica de Integración, Ámbito Salud y/o Social de la carrera Pedagogía en Educación Física, Deporte y Recreación de la Universidad Austral de Chile.

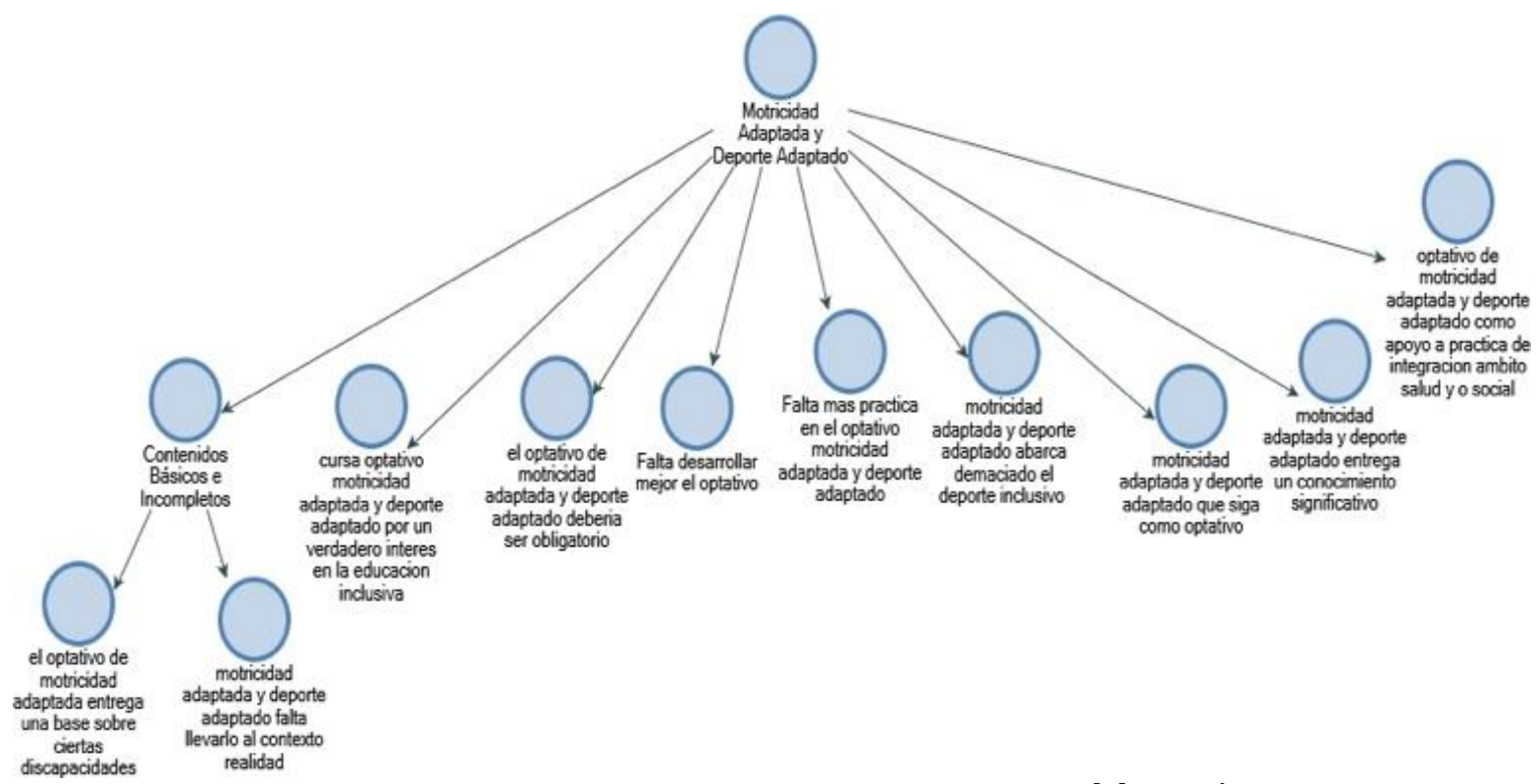

Figura 2 Pie: Motricidad Adaptada y Deporte Adaptado - Fuente: elaboración propia 
Los sujetos entrevistados se refieren al optativo de Motricidad Adaptada y Deportes Adaptados como una asignatura que entrega un conocimiento necesario para poder trabajar con personas en situación de discapacidad (sujeto 5) y que debería estar como apoyo para la asignatura de Práctica de Integración, Ámbito Salud y/o Social, puesto que en la práctica los estudiantes llegan sin saber sobre cómo trabajar con personas con NEE. "Porque empezaron con las prácticas y todos quedaron como ¡ouh! Nunca me prepararon para esto" (sujeto 8, 2017, p. 3) y los envían a los centros sin un conocimiento previo.
Por otra parte, los entrevistados/as recalcan que a pesar de que Motricidad Adaptada y Deportes Adaptados es un aporte significativo a la carrera, aún falta desarrollarla de mejor manera (sujeto 9), debido a que los contenidos son muy básicos e incompletos y se centra demasiado en Deportes Adaptados (sujetos 7, 5,3 y 2): "Yo creo que fue una asignatura que nos enseñó mucho, pero más en el ámbito del deporte competitivo" (sujeto 5, 2017, p. 3). Sería una mejor herramienta si fuese más enfocado en el desarrollo de una clase donde estén inmersos estudiantes con NEE: "podría haber sido enfocado un poco más a lo que era escolar" (sujeto 7, 2017, p. 2).

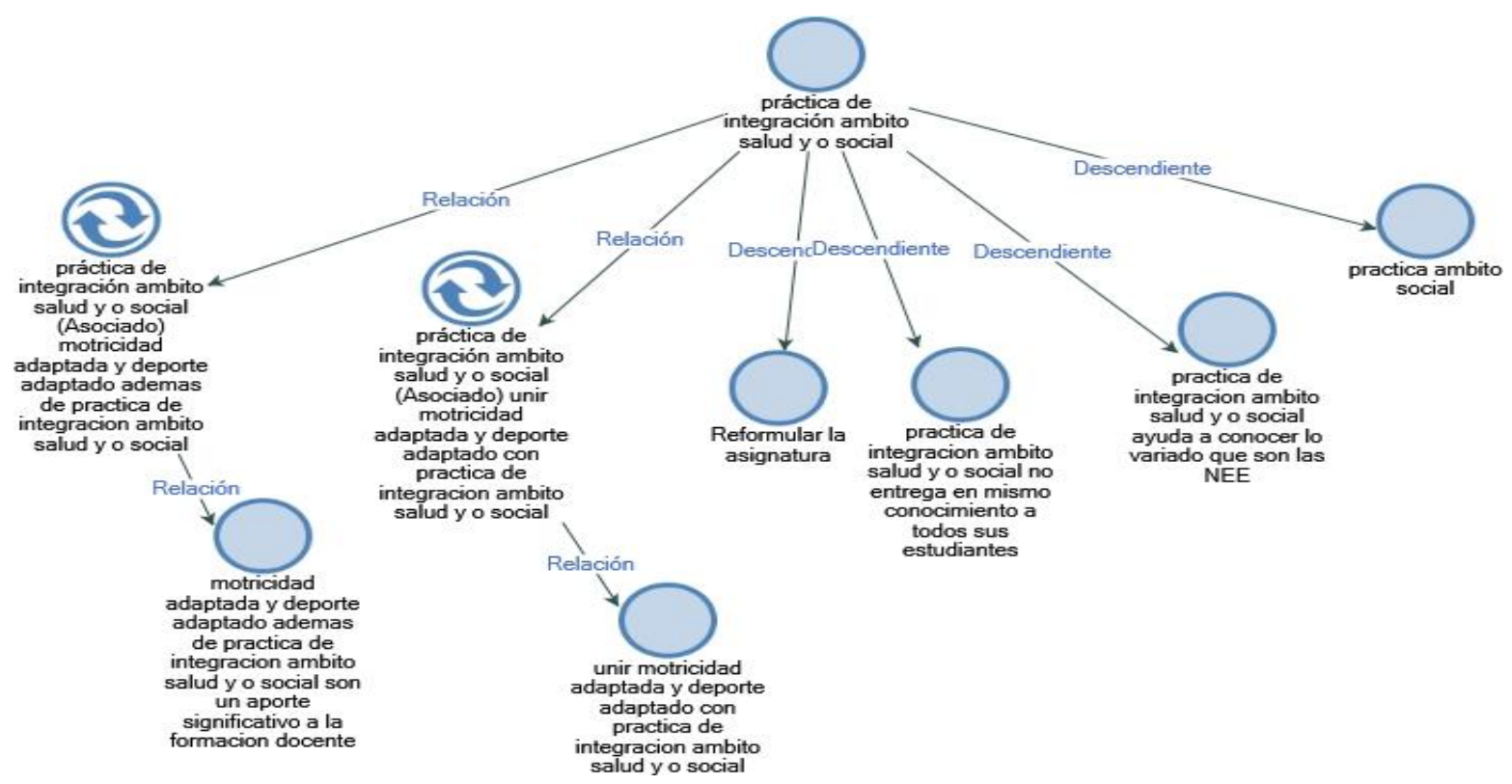

Figura 3 Pie: Práctica de Integración Ámbito salud y/o social - Fuente: Elaboración propia

Lo que respecta a la asignatura de Práctica de Integración Ámbito Salud y/o Social señalan que es un aporte significativo para la formación de estudiantes de la carrera Pedagogía en Educación 
Física, Deporte y Recreación, ya que ayuda a conocer lo variadas que son las NEE de los estudiantes. A pesar de lo anterior, suele existir el mismo comentario que los sujetos destacaron con el optativo anterior, que deberían de reformular la asignatura debido a que ésta no entrega el mismo conocimiento a todos los y las estudiantes en formación ya que algunos son enviados a centros donde suelen tratar con personas en situación de discapacidad y otras con personas en estado de integración social -temas diferentes, conocimientos diferentes. Una de las soluciones que entregan los sujetos es que unan el optativo de Motricidad Adaptada y Deportes Adaptados con Práctica de Integración ámbito Salud y/o Social.

Categorizar el conocimiento sobre inclusión e integración educativa de los estudiantes de la carrera Pedagogía en Educación Física, Deporte y recreación de la Universidad Austral de Chile.

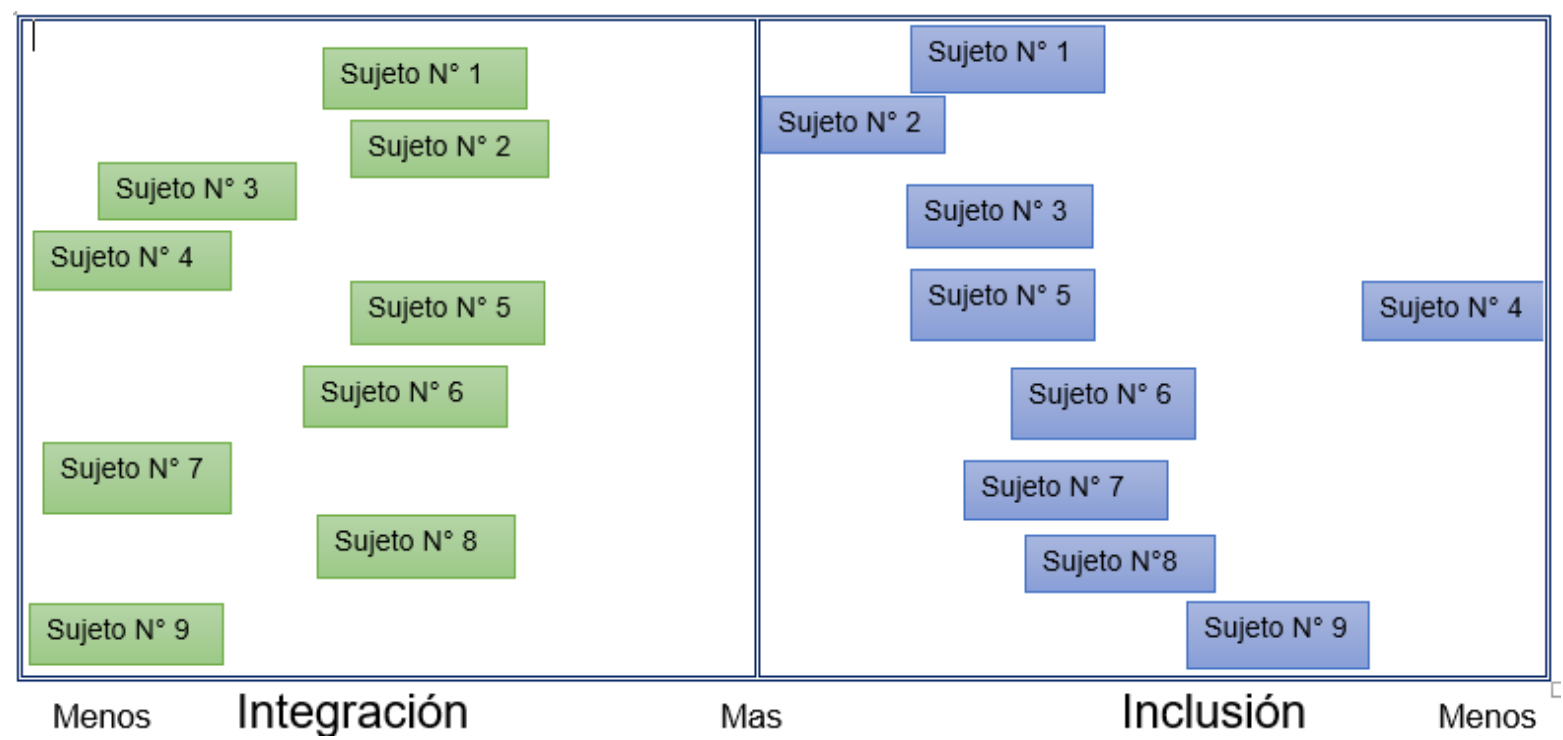

Figura 4 Pie: Conocimiento sobre Integración e Inclusión - Fuente: Elaboración propia

Lo que expresa la Figura 4 está relacionado con el conocimiento de los conceptos Inclusión e Integración que tienen los sujetos entrevistados. Quienes estén más cerca de la línea del medio son aquellos (con respecto a su lado izquierdo o derecho) que tienen mejores conocimientos sobre el concepto. Quienes estén más alejados del centro, o en el extremo de la línea lateral, expresan un desconocimiento de los temas. Por ejemplo, en el caso de la Inclusión, quien tiene un buen conocimiento sobre su concepto es el sujeto 2, pero quien tiene una definición y conocimiento erróneos es el sujeto 4.

A pesar de existir sujetos cuyos conocimientos sobre los conceptos son mínimos, en promedio se puede apreciar que más de la 
mitad tiene un conocimiento suficiente para hacer una diferencia en el desarrollo de sus clases y hacer un cambio en el establecimiento educacional donde se puedan desempeñar.

\section{Discusión}

Los resultados obtenidos reflejan un avance en la formación del profesorado en la carrera de Pedagogía en Educación Física, Deporte y Recreación de la Universidad Austral de Chile con respecto al estudio realizado por Vásquez (2013) en comparación al año 2017, donde los y las estudiantes expresan que podrían realizar una clase a personas con NEE además de conocer los conceptos sobre Inclusión e Integración. A pesar de los avances expresados, existe una inquietud por mejorar la malla curricular de la carrera, ya que esta todavía es endeble con respecto a la educación inclusiva. Aun cuando existe el optativo de Motricidad Adaptada y Deportes Adaptados no todos/as cursan por este al no ser obligatorio, lo que expresa un déficit en la formación tal como lo manifiestan Ortiz (2007) y Tenorio (2011).

También expresan su interés sobre la formación docente en conjunto con la educación inclusiva, puesto que no es cuestión de una sola persona aportar con la inclusión sino de muchos actores, y así saber dar las herramientas y apoyo para que todos en el colegio puedan aportar, como lo destacan Ainscow (2015),
Echeita (2011) y Blanco (2006) y sus escuelas inclusivas, donde todos sus integrantes ayudan con la inclusión -desde el director, pasando por el inspector general, profesores, auxiliares, etc. Aunque en algunos casos, a pesar de que existan leyes sobre inclusión e integración, los sujetos mencionan que las leyes muchas veces se quedan en el papel como lo menciona Blanco (2006).

\section{Conclusión}

La formación de profesores y profesoras en torno a la inclusión entrega mayores herramientas para poder responder a las necesidades de los y las educandos, para así poder trabajar según las capacidades de cada uno/a de ellos/as.

Con respecto a la formación de docentes en la carrera Pedagogía en Educación Física, Deporte y Recreación de la Universidad Austral de Chile en el año 2017, esta demuestra un avance en el manejo para trabajar con personas en situación de discapacidad en comparación al año 2012 según el estudio realizado por Vásquez (2013). A pesar de lo anterior, existe un vació en el estudio realizado, debido a la muestra con la que se trabajó, ya que no se tomó en consideración a la generación completa sino tan solo al grupo de estudiantes que cursaron por el optativo de Motricidad Adaptada y Deportes Adaptados, lo que provoca que no se logre saber si al menos la mitad de los estudiantes que están por 
Formación inicial de un profesorado inclusivo: avances y perspectivas en la carrera de pedagogía...

egresar cumplen con las competencias necesarias para poder responder a las necesidades educativas especiales de los estudiantes y ayudarlos para desarrollar mejor sus capacidades.

Las proyecciones que se podrían desprender de la presente investigación serían tal como las mencionan algunos de los entrevistados: reformular las asignaturas de Motricidad Adaptada y Deportes Adaptados y Práctica de Integración Ámbito Salud y/o Social para que se logre un aprendizaje significativo e igualitario en todos los docentes en formación; considerar la idea de implantar una asignatura donde se enseñe sobre diversas Discapacidades que entregue las herramientas necesarias para poder realizar una clase donde pueda participar los estudiantes en situación de discapacidad; que no solo los envíen al centro y que aprendan de la práctica, lo cual no es tan malo, para que les den las estrategias y metodologías necesarias para llegar empoderado del contexto.

\section{Referencias}

Ainscow, M. (2015). Comprendiendo el desarrollo de escuelas inclusivas. Notas y referencias bibliográficas. Extraído el, 9.

Blanco, R. (2006a). La equidad y la inclusion social: uno de los desafios de la educacion y la escuela hoy. Revista Iberoamericana sobre calidad, eficacia y cambio en educacion, 1-15.

Blanco, R. (2006b). La inclusión en educación: una cuestión de justicia y de igualdad. Sinéctica, (29).

Echeita, G. \& Ainscow, M. (2011). La educación inclusiva como derecho: marco de referencia y pautas de acción para el desarrollo de una revolución pendiente. Tejuelo: Revista de Didáctica de la Lengua y la Literatura.

Gallardo H. y Ríos J. (2013) Educación inclusiva, sus perspectivas en las orientaciones técnicas para programa de integración escolar del ministerio de educación de Chile y el proyecto educativo institucional del colegio Bernardo Felmer de Lanco, tesis para optar al título de Prof. En Ed. Física, deporte y recreación. Valdivia, Univ. Austral de Chile, Fac. Filosofía y Humanidades.

González, M. (2002). Aspectos éticos de la investigación cualitativa. Revista Iberoamericana, 85-103.

Hernández, R., Fernández, C., \& Baptista, P. (2006). Metodologia de la investigación. Mexico: McGRAW-HILL.

Infante, M. (2010). Desafios a la formacion docente: inclusion 
educativa.

Estudios

Pedagogicos, 287-297.

Mendoza, N. (2008). La formacion del profesorado en educacion fisica con relacion a las personas con discapacidad. DOSSIER, 269-279.

Ortíz, H. (2007). Formacion del profesorado y atencion a la diversidad: desafios a los procesos de innovacion curricular en educacion inicial. Revista academica $n^{\circ}$ 33, 45-60.

Schuster, A., Puente, M., Andrada, O., \& Maiza, M. (2013). La metodologia cualitativa, herramienta para investigar los fenómenos que ocurren en el aula. La investigación educativa. Revista Electronica Iberoamericana de Educación en Ciencia y Tecnologia , 109139.

Strauss, A., \& Corbin, J. (2002). Bases de la investigación cualitativa. Técnicas y procedimientos para desarrollar la teoría fundamentada. Antioquia: Universidad de Antioquia.

Tenorio, S. (2011). Formacion inicial docente y necesidades educativas especiales. Estudios Pedagogicos, 249-265.

UNESCO. (1990) Declaración mundial sobre educación para todos y Marco de acción para satisfacer las necesidades básicas de aprendizaje. Recuperado de http://www.unesco.org/educat ion/pdf/JOMTIE_S.PDF

Vasilachis de Gialdino, I., Ameigeiras, A., Chernobilsky, L., Giménez, V., Mallimaci, F., Mendizabál, N., Neiman, G., Quaranta, G. y Soneira, A. (2006). Estrategias de investigación cualitativa. Biblioteca de Educación editorial Gedisa. 1-22 pág.

Vásquez, N. (2013) “Valoración de la formación inicial docente en el ámbito de la educación inclusiva para personas con discapacidad, en los estudiantes de la carrera de pedagogía en educación física, deporte $y$ recreación de la Universidad Austral de Chile, Valdivia", tesis para optar al título de Prof. En ed. Física, deporte y recreación. Valdivia, Univ. Austral de Chile, Fac. Filosofía y Humanida 


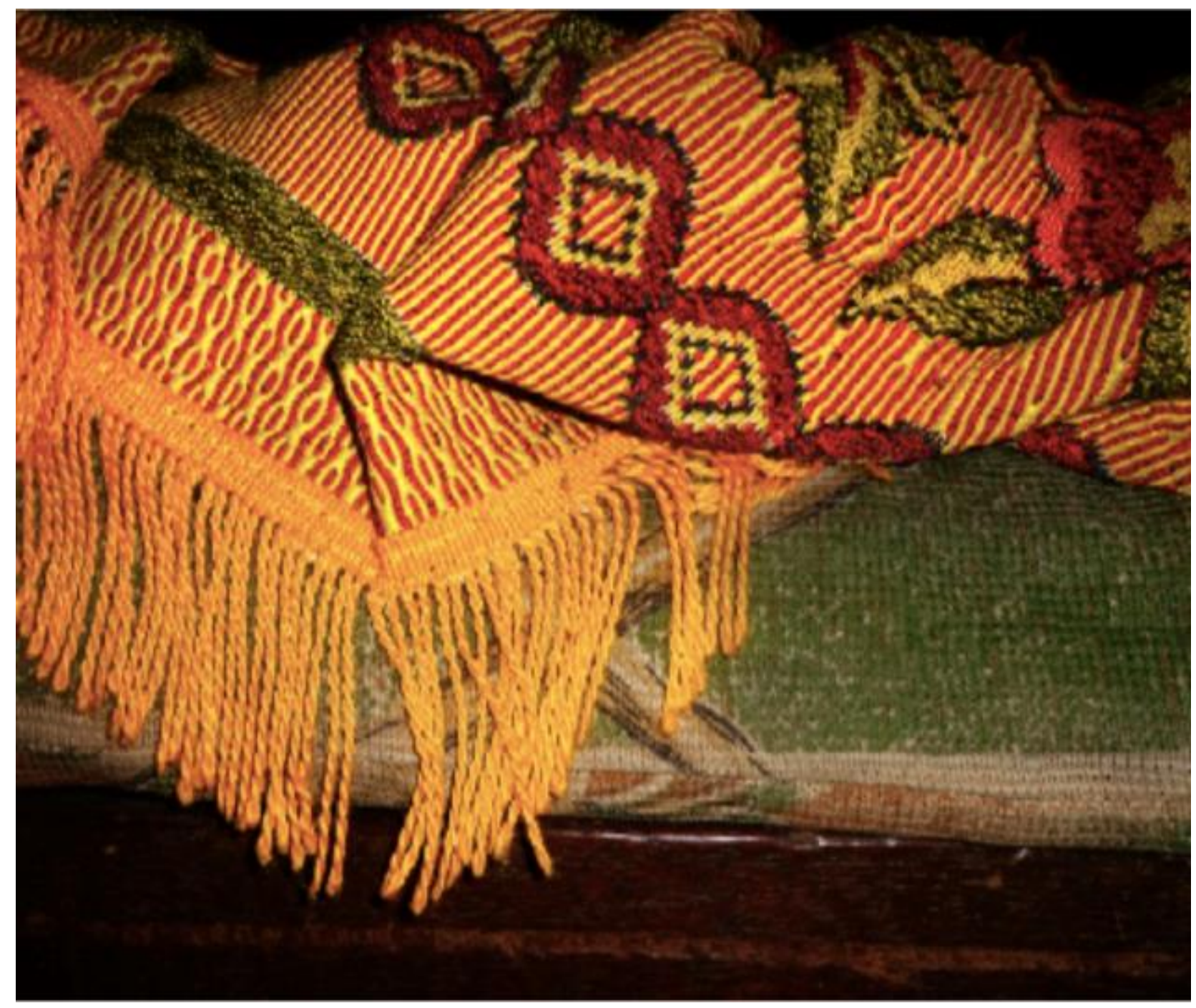

Número de la obra: 1

Título: "sobre camas"

Autor: Yenny Banesa Bonilla

Lugar: Zona rural municipio de Baraya

Finca: Los Rosales

Fecha de captura: Febrero 7 de 2014

Técnica: Fotografía digital

Fuente: Bonilla, Y. B. (2014). Sobre camas. Una mirada estética popular del municipio de Baraya. Tesis de pregrado. Universidad Surcolombiana. Neiva, 2014. p.70. 